\title{
Does Learning Curve Affect the Accuracy in Resection Alignment during Navigated Total Knee Artroplasty?
}

\author{
Yifei Dai Ph.D. ${ }^{1 *}$, Cyril Hamad Dipl. Ing. ${ }^{2}$, Amaury Jung Dipl. Ing. ${ }^{2}$, and \\ Laurent Angibaud Dipl. Ing. ${ }^{1}$ \\ ${ }^{1}$ Exactech Inc, Gainesville, FL, 32653, USA \\ ${ }^{2}$ Blue-Ortho, Gieres, 38610, FR \\ yifei.dai@exac.com
}

\begin{abstract}
This study investigated the accuracy of TKA alignment during and past learning phase for navigated total knee athroplasty. The findings demonstrated TKA during learning phase can accurately and precisely achieve surgical resection goals with no clinically meaningful compromise in alignment accuracy.
\end{abstract}

\section{Introduction}

Malalignment of the prosthesis has been shown to lead to various complications after total knee arthroplasty (TKA), such as component loosening, instability, polyethylene wear, and patellar dislocation [1-3]. It is commonly believed that $3^{\circ}$ of deviation in alignment from the mechanical axis is the threshold for reducing the risks of postoperative complications [4,5]. However, malalignment (> $3^{\circ}$ deviation) has been shown to happen in approximately one third of the patient group using conventional instrumentation [6].

Since its first introduction in TKA, computer-assisted orthopaedic surgery (CAOS) has been demonstrated to offer increased accuracy and precision to the component alignment compared to the conventional techniques [7-9]. Although most of the studies on the CAOS TKA have been focused on experienced surgeons, it is essential to understand the impact of learning on the accuracy of the surgery. The purpose of this study was to assess if learning affects the alignment of bony resection using a specific contemporary CAOS system. 


\section{Materials and Methods}

Nine surgeons were randomly selected from the technical logs of all TKA surgeries ( 7000 cases) performed between October 2012 and January 2017 using a contemporary CAOS system (ExactechGPS, Blue-Ortho, Gieres, FR). The logs did not pertain any information related to the patients. Prior to the use of the CAOS system studied, six surgeons had already experienced with CAOS TKA (experienced), while the rest three surgeons were new to the technology (novice). Each of the surgeons performed more than 80 TKAs using the CAOS system. The following surgical parameters were investigated: 1) planned resection, resection parameters defined by the surgeon prior to the bone cuts (These parameters serve as inputs for the CAOS guidance); 2) checked resection, digitalization of the realized resection surfaces by manually pressing an instrumented checker onto the bony cuts. Due to that anterior, posterior and chamfer cuts of the femur were all corresponded to the distal resection, only the distal resection was evaluated for the femur.

Deviations in the alignment between planned and checked resections were calculated in coronal and sagittal planes for both tibia and femur (planned vs. checked). The deviations were compared between the first 20 cases (in learning curve) and the last 20 cases (well past learning curve) within each surgeon. The selection of 20 cases as the length of the learning stage was based on a previous study on the specific CAOS system investigated [10]. Any significance detected $(\mathrm{p}<0.05)$ with greater than $1^{\circ}$ difference in means indicated clinically meaningful impact on resection alignment by the learning phase. A resection was determined as acceptable if the deviation was less than $3^{\circ}$ from the planned resection.

\section{Results}

For pooled data, no significant difference was found between the first 20 cases and the last 20 cases from the experienced surgeons (N.S.) (Table 1A). Although some significance were found in the novice surgeon, the differences were not clinically meaningful (difference in means were no more than $0.4^{\circ}$, Table $1 \mathrm{~A}$ ). The surgeon-specific results exhibit some significant differences between the first 20 and the last 20 TKA cases for both experienced and novice surgeons (Table 2). However, none of the significances were clinically meaningful, as the differences in means were no more than $0.7^{\circ}$ (Table 2). The resections in both the first 20 cases and the last 20 cases demonstrated acceptable rates of over 95\% in alignment for both experienced and novice surgeons (Table 1B).

\section{Discussion}

This study demonstrated that independent of the surgeon's prior CAOS experiences, the CAOS system investigated can provide an accurate and precise solution to assist in achieving surgical resection goals with no clinically meaningful compromise in alignment accuracy during the learning phase. Compared to studies that have reported that only $70-80 \%$ of the TKA cases can achieve satisfactory lower limb alignment (within $\pm 3^{\circ}$ of varus/valgus relative to the mechanical axis) [11,12], the results showed that CAOS TKA substantially minimized alignment outliers, independent of whether the surgeon was in or well past the learning curve. Several studies have been published regarding CAOS TKA learning curve by comparing the accuracy between experienced surgeons/centres and beginner surgeons/centres $[13,14]$. This study performed assessment within the same surgeon during and past learning curve to shed a light on the progress of alignment accuracy. The applied methodology may improve the understanding of the impact of learning curve by excluding the inter-surgeon differences. 


\section{References}

[1] Jeffery RS, et al. Coronal alignment after total knee replacement. J Bone Joint Surg Br,73:709$14,1991$.

[2] Wasiliewski RC, et al. Wear patterns on retrieved polyethylene tibial inserts and their relationship to technical considerations during total knee arthroplasty. Clin Orthop Relat Res,299:31-43,1994.

[3] Ritter MA, et al. Postoperative alignment of total knee replacement: its effect on survival. Clin Orthop Relat Res,299:153-6,1994.

[4] Jeffery RS, et al. Coronal alignment after total knee replacement. J Bone Joint Surg Br,73(5):709$14,1991$.

[5] Insall JN. Presidential address to The Knee Society. Choices and compromises in total knee arthroplasty. Clin Orthop Relat Res,226:43-8,1998.

[6] Cheng T, et al. Does computer-assisted surgery improve postoperative leg alignment and implant positioning following total knee arthroplasty? A meta-analysis of randomized controlled trials? Knee Surg Sports Traumatol Arthrosc,20(7):1307-22,2012.

[7] Blakeney WG, et al. Computer-assisted techniques versus conventional guides for component alignment in total knee arthroplasty: a randomized controlled trial. J Bone Joint Surg Am,93(15):1377-84,2011.

[8] Shinozaki T, et al. Computer-assisted total knee arthroplasty: comparisons with the conventional technique. Kurume Med J,58(1):21-6,2011.

[9] Chen JY, et al. Less outliers in pinless navigation compared with conventional surgery in total knee arthroplasty. Knee Surg Sports Traumatol Arthrosc,22(8):1827-32,2004.

[10] Petrera P, et al. ExactechGPS Guidance System Does Not Increase Operative Time When Compared to Conventionally Instrumented Total Knee Arthroplasty. CAOS 2015.

[10] Hetaimish BM, et al. Meta-analysis of navigation vs conventional total knee arthroplasty. J Arthroplasty,27(6):1177-82,2012.

[11] Ensini A, et al. Alignments and clinical results in conventional and navigated total knee arthroplasty. Clin Orthop Relat Res,457:156-62,2007.

[12] Jenny JY, et al. Learning curve in navigated total knee replacement. A multi-centre study comparing experienced and beginner centres. knee,15:80-4,2008.

[13] Manzotti A, et al. Relationship between cutting errors and learning curve in computer-assisted total knee replacement. Int Orthop,34(5):655-62.

\section{Disclosures}

LA and YD are employees of Exactech Inc; AJ and CH are employees of Blue Ortho. 


\begin{tabular}{|c|c|c|c|c|c|}
\hline $\begin{array}{l}\text { Prior CAOS } \\
\text { experience }\end{array}$ & Pooled & $\begin{array}{c}\text { Tibial } \\
\operatorname{var} / v a l\left({ }^{\circ}\right)\end{array}$ & $\begin{array}{c}\text { Tibial posterior } \\
\text { slope }\left(\left(^{\circ}\right)\right.\end{array}$ & $\begin{array}{c}\text { Femoral } \\
\operatorname{var} / v a l \\
\left({ }^{\circ}\right)\end{array}$ & $\begin{array}{c}\text { Femoral } \\
\text { flex/ext }\left({ }^{\circ}\right)\end{array}$ \\
\hline \multirow{3}{*}{ Experienced } & First 20 cases & $0.7 \pm 0.6$ & $0.9 \pm 0.7$ & $0.7 \pm 0.7$ & $1.0 \pm 0.9$ \\
\hline & Last 20 cases & $0.7 \pm 0.5$ & $1.0 \pm 0.9$ & $0.6 \pm 0.6$ & $1.0 \pm 0.7$ \\
\hline & $p$ & 0.404 & 0.169 & 0.347 & 0.895 \\
\hline \multirow{3}{*}{ Novice } & First 20 cases & $0.7 \pm 0.4$ & $1.2 \pm 1.0$ & $0.7 \pm 1.1$ & $1.0 \pm 0.8$ \\
\hline & Last 20 cases & $0.5 \pm 0.4$ & $0.8 \pm 0.6$ & $0.4 \pm 0.4$ & $0.8 \pm 0.5$ \\
\hline & $\mathrm{p}$ & 0.031 & 0.019 & 0.067 & 0.197 \\
\hline \multicolumn{6}{|l|}{ B } \\
\hline $\begin{array}{l}\text { Prior CAOS } \\
\text { experience }\end{array}$ & $\begin{array}{c}\text { Pooled } \\
\% \text { acceptable } \\
\text { resections }\end{array}$ & $\begin{array}{l}\text { Tibial } \\
\text { var/val }\end{array}$ & $\begin{array}{c}\text { Tibial } \\
\text { posterior slope }\end{array}$ & $\begin{array}{l}\text { Femoral } \\
\text { var/val }\end{array}$ & $\begin{array}{l}\text { Femoral } \\
\text { flex/ext }\end{array}$ \\
\hline \multirow{2}{*}{ Experienced } & First 20 cases & $99.1 \%$ & $98.2 \%$ & $97.4 \%$ & $97.4 \%$ \\
\hline & Last 20 cases & $100.0 \%$ & $95.7 \%$ & $99.1 \%$ & $98.2 \%$ \\
\hline \multirow{2}{*}{ Novice } & First 20 cases & $100.0 \%$ & $96.7 \%$ & $96.7 \%$ & $98.4 \%$ \\
\hline & Last 20 cases & $100.0 \%$ & $98.4 \%$ & $100.0 \%$ & $100.0 \%$ \\
\hline
\end{tabular}

Table 1: A) Pooled alignment deviations (planned vs checked) for the first 20 cases group and the last 20 cases group. Significant differences between the first 20 cases and the last 20 cases were marked as red. None of the significant differences found were clinically important (differences in means no more than $0.4^{\circ}$ ). B) Percentages of acceptable resections for the first 20 cases group and the last 20 cases group. 


\begin{tabular}{|c|c|c|c|c|c|}
\hline $\begin{array}{l}\text { Experienced } \\
\text { surgeon }\end{array}$ & & $\begin{array}{c}\text { Tibial } \\
\text { var/val }\left({ }^{\circ}\right)\end{array}$ & $\begin{array}{c}\text { Tibial posterior } \\
\text { slope }\left({ }^{\circ}\right)\end{array}$ & $\begin{array}{c}\text { Femoral } \\
\text { var/val }\left({ }^{\circ}\right)\end{array}$ & $\begin{array}{l}\text { Femoral } \\
\text { flex/ext }\left({ }^{\circ}\right)\end{array}$ \\
\hline & First 20 cases & $0.9 \pm 0.6$ & $0.6 \pm 0.4$ & $0.7 \pm 1.1$ & $0.9 \pm 0.7$ \\
\hline \multirow[t]{3}{*}{$\# 1$} & Last 20 cases & $1.0 \pm 0.5$ & $0.5 \pm 0.4$ & $0.5 \pm 0.4$ & $0.6 \pm 0.6$ \\
\hline & $\mathrm{p}$ & 0.490 & 0.299 & 0.363 & 0.129 \\
\hline & First 20 cases & $0.6 \pm 0.6$ & $0.8 \pm 0.7$ & $0.6 \pm 0.4$ & $0.9 \pm 0.6$ \\
\hline \multirow[t]{3}{*}{$\# 2$} & Last 20 cases & $0.7 \pm 0.5$ & $1.0 \pm 0.8$ & $0.8 \pm 0.6$ & $1.1 \pm 0.9$ \\
\hline & $\mathrm{p}$ & 0.485 & 0.452 & 0.263 & 0.398 \\
\hline & First 20 cases & $1.0 \pm 1.1$ & $0.8 \pm 0.7$ & $0.8 \pm 0.6$ & $1.3 \pm 0.9$ \\
\hline \multirow[t]{3}{*}{$\# 3$} & Last 20 cases & $0.3 \pm 0.1$ & $1.0 \pm 0.9$ & $0.4 \pm 0.3$ & $0.9 \pm 0.5$ \\
\hline & $\mathrm{p}$ & 0.008 & 0.657 & 0.020 & 0.133 \\
\hline & First 20 cases & $0.6 \pm 0.5$ & $1.2 \pm 0.8$ & $1.1 \pm 1.0$ & $1.0 \pm 1.1$ \\
\hline \multirow[t]{3}{*}{$\# 4$} & Last 20 cases & $1.0 \pm 0.5$ & $1.2 \pm 1.2$ & $1.1 \pm 0.9$ & $1.1 \pm 0.8$ \\
\hline & $\mathrm{p}$ & 0.038 & 0.977 & 0.988 & 0.762 \\
\hline & First 20 cases & $0.5 \pm 0.4$ & $1.2 \pm 0.8$ & $0.7 \pm 0.5$ & $1.0 \pm 0.7$ \\
\hline \multirow[t]{3}{*}{ \#5 } & Last 20 cases & $0.8 \pm 0.4$ & $1.2 \pm 1.1$ & $0.5 \pm 0.3$ & $0.8 \pm 0.7$ \\
\hline & $\mathrm{p}$ & 0.076 & 0.614 & 0.121 & 0.891 \\
\hline & First 20 cases & $0.5 \pm 0.3$ & $0.7 \pm 0.6$ & $0.5 \pm 0.5$ & $0.9 \pm 1.2$ \\
\hline \multirow[t]{2}{*}{ \#6 } & Last 20 cases & $0.6 \pm 0.4$ & $1.2 \pm 0.8$ & $0.7 \pm 0.5$ & $1.2 \pm 0.8$ \\
\hline & $\mathrm{p}$ & 0.316 & 0.022 & 0.307 & 0.324 \\
\hline \multicolumn{6}{|l|}{ B } \\
\hline \multirow[t]{2}{*}{$\begin{array}{l}\text { Novice } \\
\text { surgeon }\end{array}$} & & $\begin{array}{c}\text { Tibial } \\
\text { var/val }\left({ }^{\circ}\right) \\
\end{array}$ & $\begin{array}{l}\text { Tibial posterior } \\
\text { slope }\left({ }^{\circ}\right)\end{array}$ & $\begin{array}{c}\text { Femoral } \\
\operatorname{var} / v a l \\
\left({ }^{\circ}\right)\end{array}$ & $\begin{array}{c}\text { Femoral } \\
\text { flex/ext }\left({ }^{\circ}\right)\end{array}$ \\
\hline & First 20 cases & $0.5 \pm 0.6$ & $0.6 \pm 0.5$ & $0.6 \pm 0.5$ & $1.2 \pm 0.8$ \\
\hline \multirow[t]{3}{*}{$\# 1$} & Last 20 cases & $0.4 \pm 0.4$ & $0.8 \pm 0.6$ & $0.3 \pm 0.2$ & $1.0 \pm 0.4$ \\
\hline & $\mathrm{p}$ & 0.406 & 0.620 & 0.026 & 0.430 \\
\hline & First 20 cases & $0.6 \pm 0.5$ & $1.1 \pm 1.0$ & $0.8 \pm 1.6$ & $1.1 \pm 1.0$ \\
\hline \multirow[t]{3}{*}{ \#2 } & Last 20 cases & $0.3 \pm 0.2$ & $1.0 \pm 0.7$ & $0.4 \pm 0.4$ & $0.6 \pm 0.5$ \\
\hline & $\mathrm{p}$ & 0.018 & 0.627 & 0.235 & 0.048 \\
\hline & First 20 cases & $0.8 \pm 0.6$ & $1.5 \pm 1.0$ & $0.7 \pm 0.6$ & $0.8 \pm 0.6$ \\
\hline \multirow[t]{2}{*}{$\# 3$} & Last 20 cases & $0.9 \pm 0.6$ & $0.8 \pm 0.6$ & $0.7 \pm 0.4$ & $0.9 \pm 0.6$ \\
\hline & $\mathrm{p}$ & 0.841 & 0.013 & 0.955 & 0.841 \\
\hline
\end{tabular}

Table 2: Surgeon-specific alignment deviations (planned vs checked) for the first 20 cases group and the last 20 cases group. Significant differences between the first 20 cases and the last 20 cases were marked as red. None of the significant differences found were clinically important (differences in means no more than $0.7^{\circ}$ ). 